\title{
Rotatory quantization of charge-conjugation symmetric systems. 3. Relativistic fields.
}

\author{
Zahid Zakir $^{1}$
}

\begin{abstract}
Quantum theory of complex fields with rotational modes based on a harmonic rotator model is constructed. For purely rotational modes the energy spectrum is equidistant, observables are automatically normal-ordered and there is no zero-point vacuum energy and zero-point charge. Frequencies of quanta are angular speeds of rotating field vectors (in real or field spaces). States of two signs of the helicity (particle-antiparticle) are related through the crossing symmetry. The well-known examples are photon field with circular polarization and complex fields. The spin and isospins of particles appear as related to their frequencies, representing angular momenta of rotations of field vectors with these frequencies. It is shown that the standard covariant perturbation theory is constructed in fact for description on the basis of harmonic rotators, where the recipe of transition from oscillatory to the rotatory representations of modes earlier it has been found empirically as normal-ordering of operators. The vacuum energy vanishes for free Hamiltonians and C-symmetric interactions.
\end{abstract}

PACS: 03.70. + k, 11.30.Er

Key words: quantization of fields, charge conjugation, parity, vacuum energy

\section{Содержание}

Introduction.

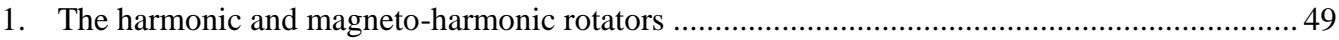

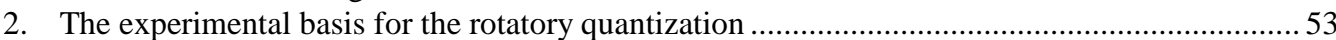

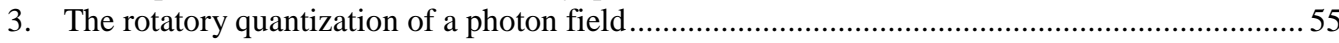

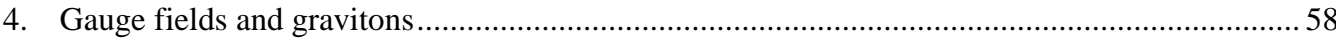

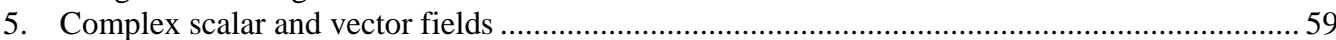

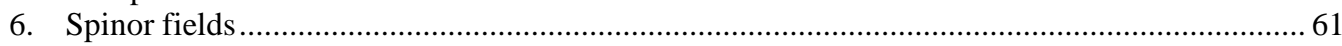

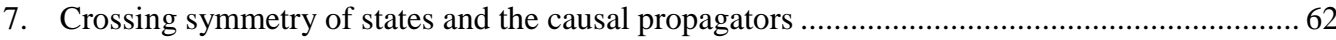

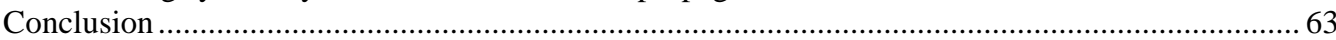

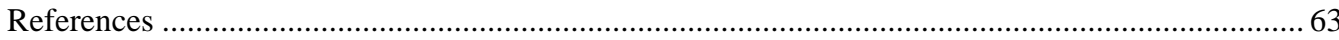

\section{Introduction}

The standard formulation of quantum theory of relativistic fields is based on oscillatory quantization, i.e. on a hypothesis about a vibrational nature of fluctuations of fields (oscillatory postulate). But, the field theory can be based on the linear harmonic oscillator model only if the zero-point energy of modes are exactly cancelled. If there is no such cancellation, the vacuum energy divergence, due to the existence of gravitation, makes such field theory as inconsistent.

For compensation of this inadequacy the theory has been completed by a hypothesis of normal ordering, which in fact is a hidden rejection of the oscillatory postulate. As the result, it had led to long and unsuccessful searches of a physical basis for the normal ordering at preserving the oscillatory postulate.

\footnotetext{
${ }^{1}$ Centre for Theoretical Physics and Astrophyics, Tashkent, Uzbekistan; zahidzakir@theor-phys.org
} 
Another way for the solution of the problem is, instead of starting from the hypothesis about vibrational fluctuations with rejection of its inevitable consequence, to raise the question about searching an alternative to the harmonic oscillator model in which the energy spectrum would be also equidistant and linear under frequency, but which would not contain a zero-point energy.

A way to such more adequate physical treatment of fluctuations of fields suggests an example of photons, pure states of which are states with circular polarization. Such photons are emitted by a rotating dipole when transverse field vectors are constant in modulo and rotate around photon's momentum direction, the energy levels are equidistant. There frequency is angular speed of rotation of field vectors, photon's spin is an angular momentum and a helicity plays a role of a conserved chiral charge.

All these exactly correspond to properties of a harmonic rotator model with purely rotational modes [1,2], in which the energy spectrum is equidistant, linear under frequency and does not contain a zero-point energy, so that the normal ordering appears naturally. This model, unusual for the nonrelativistic systems, where a radial mode presents always, at least in the form of zero-point fluctuations, but it is perhaps only adequate one for the relativistic fields where purely rotational modes are admissible.

All relativistic fields, corresponding to observed fundamental particles, are complex ones because of helicity states and presence of antiparticles, and their quanta in pure states are emitted by sources transferring them an angular momentum, spin or charge. A zero-point energy of their vacuum does not observed up to now in any experiment, and those effects to which refer for "proving" the existence of zero-point fluctuations, in fact are fully explained by influences of real sources and there no room for the contributions of the zero-point vacuum fluctuations [3].

For these reasons, in the present paper the theory of relativistic fields is formulated on the basis of the harmonic rotator model by interpreting the frequencies of quanta as the angular speeds of rotation of field vectors in real or isotopic spaces. Radial fluctuations in complex field space are excluded by means of requirements of basic discrete symmetries. In such theory the observables are normal ordered automatically and the vacuum energy does not arise due to the fundamental physical reason.

In the Section 1 main facts of the harmonic rotator theory are presented and in Section 2 the experimental bases for the rotatory quantization are discussed. In sections 35 quantization of a field of photons, a charged scalar and vector fields and a spinor field are formulated. In section 6 rotatory interpretation of spins and isospins of fields is presented. In section 7 quantization of weak gauge fields and a graviton field is considered, and in section 8 commutators and causal propagators for rotatory modes of fields, and a crossing symmetry between right- and left-rotating modes are considered.

\section{The harmonic and magneto-harmonic rotators}

At rotatory quantization of relativistic fields, which will be developed in the present paper, instead of the hypothesis about vibrations, we will start from following physical fact. For quanta emitted by sources rotating with a circular frequency $\omega$, a frequency in their energy corresponds to a rotation frequency $\omega$ of field vectors around a momentum direction at a constant modulus of field vectors. In this case quanta of normal modes of fields are similar to quanta of the harmonic rotator $[1,2]$. Further we present a short review of main facts of the harmonic rotator theory for further using at quantization of fields. 
Let a particle of mass $m$ moves in the harmonic potential on a plane $(x, y)$. It is accepted to name this system as a planar harmonic oscillator. The Lagrangian and a Hamiltonian in polar coordinates $(r, \theta)$, where $r^{2}=x^{2}+y^{2}$, have the form:

$$
\begin{gathered}
L=\frac{m}{2} \dot{r}^{2}+r^{2} \frac{m}{2}\left(\dot{\theta}^{2}-\omega^{2}\right), \\
H=p_{r} \dot{r}+M \dot{\theta}-L=\frac{1}{2 m}\left(p_{r}^{2}+\frac{M^{2}}{r^{2}}+m^{2} \omega^{2} r^{2}\right),
\end{gathered}
$$

with angular momentum $M= \pm m r^{2} \dot{\theta}$. In a particular case, when $M=0$, the particle makes purely radial vibrations as a linear harmonic oscillator.

In other particular case of such system when the angular momentum is non-zero $M \neq 0$, there appear qualitatively new physical properties related with symmetry under two rotation directions and angular momentum conservation. Really, because of increase of centrifugal force the particle cannot transit through center, and also there is a two-fold degeneration of levels that leads to a new discrete symmetry. All these are more peculiar to rotators than oscillators and it is suitable to name such system as a vibrating harmonic rotator.

Notice, that the transition from a circular harmonic oscillator to a particular case of the vibrating harmonic rotator supposes $M \neq 0$, but this requirement is not fulfilled for a ground state. Therefore, taking into account that rotational modes do not contribute to ground state energy, this energy is equal or to energy of zero-point radial vibrations, or vanishes if the radial mode is excluded exactly.

For us it is most interesting this last special case of the vibrating harmonic rotator when the radial vibrations fully absent. This system is a harmonic rotator - a particle, moving in the planar harmonic potential, kinetic energy of which is reduced to purely rotational energy $M^{2} / 2 m r^{2}$. In the classical theory it can be obtained from the vibrating harmonic rotator by two ways - or by introducing a constraint vanishing a radial momentum, or by using symmetry properties [1,2]. In both cases there no radial momentum contributions to energy and at quantization the system does not contain radial excitations and a radial zero-point energy.

More simple and consecutive way, which nevertheless leads to the same physical results, consist in constructing of the harmonic rotator model not as planar oscillator's special case, but as a self-consistent and fundamental model, alternative to the linear harmonic oscillator model. For this purpose from the very beginning we will introduce a model Lagrangian where the kinetic term is reduced only to the rotational energy. The Lagrangian and the Hamiltonian of the harmonic rotator at such approach have the form:

$$
\begin{gathered}
L_{0}=r^{2} \frac{m}{2}\left(\dot{\theta}^{2}-\omega^{2}\right), \\
H_{0}=M \dot{\theta}-L_{0}=\frac{1}{2 m}\left(\frac{M^{2}}{r^{2}}+m^{2} \omega^{2} r^{2}\right)=\left.M \omega\right|_{\dot{\theta}= \pm \omega} .
\end{gathered}
$$

Here the radial coordinate (more exactly $r^{2}$ ) is in fact a Lagrange multiplier for which, as it is known, a momentum should not be introduced by definition.

Thus, energy of the harmonic rotator is proportional to the rotation frequency $\omega$, which is constant, and to the angular momentum $M_{n_{\theta}}= \pm m r_{n_{\theta}}^{2} \omega=n_{\theta} \hbar$, which is 
quantized with $n_{\theta}=0, \pm 1, \ldots$ The energy spectrum appears as equidistant, linear on rotation frequency, two fold degenerate and beginning from zero:

$$
E_{n, s}=\hbar \omega\left|n_{\theta}\right| \text {. }
$$

The standard and widely applying physical realization of the harmonic rotator is motion of a charged particle in a constant uniform magnetic field $H_{z}$ (see [1,2]). In the classical theory the particle moves along strictly circular orbits on the plane $(x, y)$, and the magnetic field acts as a harmonic potential on this rotation plane. Thus, the equation of motion for the radial degree of freedom is trivial $\dot{r}=0$ and leads to vanishing of the radial momentum, consequently, there are no radial excitations in the system and it is not need to exclude them specially. But, quantization at presence of the radial degree of freedom as a dynamical one leads to a zero-point energy of radial vibrations and then to the breaking of a charge conjugation symmetry of the system [2].

For this reason, if at quantization we want to preserve the exact charge conjugation symmetry of the classical system, we can restrict ourselves by purely rotational modes, which by definition do not contain the zero-point energy. The Lagrangian of such magneto-harmonic rotator can be chosen in two forms - or we start with a standard classical Lagrangian

$$
L_{\perp}=\frac{m}{2} \dot{r}^{2}+r^{2} \frac{m}{2}\left[\left(\dot{\theta}+\omega_{ \pm}\right)^{2}-\omega_{ \pm}^{2}\right],
$$

and introduce the charge conjugation symmetry requirements, or we choose such kinetic term in which the radial momentum does not figure initially:

$$
L_{0}=r^{2} \frac{m}{2}\left[\left(\dot{\theta}+\omega_{ \pm}\right)^{2}-\omega_{ \pm}^{2}\right] .
$$

The Lagrangian (7) differs from the harmonic rotator's Lagrangian (3) only by angular speed's shift: $\dot{\theta} \rightarrow \dot{\theta}+\omega_{ \pm}$. To the energy levels of the magneto-harmonic rotator there contribute only purely rotational modes of the harmonic rotator:

$$
E_{n_{\theta}}=2 \omega\left(\left|n_{\theta}\right|-n_{\theta}\right)=\left\{\begin{array}{cc}
\omega_{H}\left|n_{\theta}\right|, & n_{\theta}<0, \\
0, & n_{\theta}>0 .
\end{array}\right.
$$

Here the cyclotron frequency $\omega_{H}=e\left|H_{z}\right| / m_{0} c$ is twice more than harmonic rotator's frequency: $\omega_{H}=2 \omega_{ \pm}$and the radii of levels are less in $\sqrt{2}$ times. Thus, in the magnetoharmonic rotator model with the Lagrangian (7) the spectrum begins from zero. The standard choice (6) for the Lagrangian will be useful at introduction of complex variables and it will be considered below.

In the two above presented physical examples the rotational contributions have been separated from the radial ones, which allowed us to choose the model Lagrangians without the radial kinetic term. In a general case, when there is no such explicit separation, the problem consists in searching of physical mechanisms allowing one to leave only purely rotational modes. As it will be shown in [2], in terms of complex variables, suitable for relativistic fields, the role of such physical mechanism play the charge conjugation symmetry and other discrete symmetries of states.

At quantization of the magneto-harmonic rotator in complex variables the complex conjugate velocities on the rotation plane any more do not commute: $\left[\dot{q}, \dot{q}^{*}\right]=2 \omega / m$. For this reason there are two kinds of Lagrangians with different 
orderings of products of these velocities, which in the classical case transfer into the standard classical Lagrangian (6) and, consequently, intuitively have been considered as equivalent ones:

$$
\begin{gathered}
L_{0}=m \dot{q}^{*} \dot{q}-m \omega \cdot i\left(q^{*} \dot{q}-\dot{q}^{*} q\right), \\
L_{\perp}=\frac{1}{2} m\left(\dot{q}^{*} \dot{q}+\dot{q} \dot{q}^{*}\right)-m \omega \cdot i\left(q^{*} \dot{q}-\dot{q}^{*} q\right) .
\end{gathered}
$$

Taking into account of this fact, the Lagrangians (9) - (10) we can rewrite in the forms:

$$
\begin{gathered}
L_{0}=m\left[\left(D_{t} q\right)^{*}\left(D_{t} q\right)-\omega^{2} q^{*} q\right] . \\
L_{\perp}=m\left[\left(D_{t} q\right)^{*}\left(D_{t} q\right)-\omega^{2} q^{*} q\right]-\omega,
\end{gathered}
$$

where the "extended" time derivative $D_{t}=\partial_{t}+i \omega$ is introduced. Unlike the velocities, the "extended" velocities $D_{t} q$ and $\left(D_{t} q\right)^{*}$, which are momenta up to a coefficient (mass), commutate among themselves. The Lagrangians (11)-(12) then lead to the Hamiltonians:

$$
\begin{gathered}
H_{0}=\frac{1}{m}\left(p+i m \omega q^{*}\right)\left(p^{*}-i m \omega q\right)=2 \omega a^{*} a=\omega_{H} Q, \\
H_{\perp}=\frac{1}{m}\left(p+i m \omega q^{*}\right)\left(p^{*}-i m \omega q\right)+\omega=2 \omega a^{*} a+\omega=\omega_{H}\left(Q+\frac{1}{2}\right),
\end{gathered}
$$

Thus, the "minimal" Lagrangian $L_{0}$ leads to the "minimal" Hamiltonian $H_{0}$ without the zero-point energy and, consequently, this system is a magneto-harmonic rotator. From the symmetrized Lagrangian $L_{\perp}$ follows the symmetrized Hamiltonian $H_{\perp}$ with the standard spectrum, containing the energy $E_{(0)}=\omega_{H} / 2$ of the radial zero-point fluctuations (transformed by the magnetic field to random motion along arc-wise paths).

The states of the magneto-harmonic rotator with $Q$ are defined so that corresponds to rotations of a particle with a positive charge, frequency and energy. A charge conjugate particle rotates in opposite direction, but after the charge conjugation with inversion of $z$ axis we obtain the states of the former particle. Thus, the discrete symmetries allow to express the operators $b^{+}, b$ of a charge-conjugate state through the operators $a^{+}, a$ and to write the observables of the systems in the form:

$$
\begin{gathered}
Q=\tilde{Q}+\tilde{Q}^{c}=a^{+} a-b^{+} b, \\
H_{0}=\omega_{H} Q=\omega_{H}\left(a^{+} a+b^{+} b\right) .
\end{gathered}
$$

Then, by comparison with (13)-(14) we come to the same relations between the charge operators, as in the case of the harmonic rotator [2].

Thus, in rotating systems with charge conjugation symmetry the charge operator figures instead of the angular momentum. As earlier it has been shown [2], at choosing a Lagrangian in the "minimal" form this symmetry naturally excludes the zero-point energy and the zero-point charge, and at quantization such systems are reduced to a set of harmonic rotators.

A model of a rotating chain of the harmonic rotators, considered in [2], allows us in a (formally) continuous limit turn to fields, normal modes of which behave as the harmonic rotators. In this model the doubled chain of the bounded particles forming the 
harmonic rotators, arranging along a common axis $z$ with a step $a$ is considered. At synchronous rotations of rotators on the planes $\left(x, y, z_{i}\right)$ to adding (or removing) a quantum of angular momentum to one of rotators leads to propagation of a transverse wave along $z$ axis. At periodic perturbations the frequency decomposition gives normal modes with a wave number $k_{z}$ and frequency $\omega_{k}$. There the frequency $\omega_{0}$, by which the total chain rotates, appears as a "rest mass" $m_{0}=\omega_{0}=$ const of quanta propagating along the chain.

\section{The experimental basis for the rotatory quantization}

Since the presence or lack of the zero-point fluctuations for any system can be checked up experimentally, we will begin with the experimental bases for the rotatory quantization (references to the literature see in [3]).

In particle physics well known observables effects - the Lamb shift, the anomalous magnet moment and the Casimir effect - have been described as effects of quantum fluctuations of fields of real sources and considered as proofs of the existence of such fluctuations. At the same time the same effects have been accepted to treat and widely popularize as the proofs of a reality of the zero-point fluctuations of vacuum fields without any source $[4,5]$.

Such contradictory character of the situation did not find an explanation in the frameworks of the oscillatory treatment of the fluctuations of fields. The intuitive explanations that they are two manifestations of the same picture and that there is no contradiction, are prevalent illusions and in fact are inadequate the real situation.

Really, in the oscillatory treatment with the energy of zero-point fluctuations of electromagnetic field's vacuum fields are related the field strengths $\mathbf{E}_{(0)}, \mathbf{B}_{(0)}$ :

$$
H_{0}^{(0)}=2 \int d^{3} k \frac{1}{2} \omega_{k}=\int d^{3} x \frac{1}{2}\left(\mathbf{E}_{(0)}^{2}+\mathbf{H}_{(0)}^{2}\right)
$$

and in the total Hamiltonian it inevitably contributes an electrostatic energy of charges with these vacuum fields $H_{I}^{(0)}=\mathbf{j} \cdot \mathbf{A}_{(0)}$, where $\mathbf{A}_{(0)}$ is a potential corresponding to $\mathbf{E}_{(0)}, \mathbf{B}_{(0)}$. Energy of interaction with vacuum fields $H_{I}^{(0)}$ enters into the Hamiltonian additively with energy of interaction with the real sources $H_{I}$, and thus both kinds of contributions should be summed. Shifts of energy, for example, practically will be doubled:

$$
\Delta E_{I}=\Delta E_{I}^{(0)}+\Delta E_{I}^{r} \approx 2 \Delta E_{I}^{r} .
$$

The reluctance to sum of these contributions by followers of the vibrational treatment aroused by two reasons. From one hand, in the oscillatory treatment it is impossible to explain such explicit and catastrophic disagreement between the theory and experiment. On the other hand, even those who, after a detailed and open-minded analysis, come to a direct and clear conclusion about lack of any evidence for the zeropoint fluctuations, doubted on possibility to formulate quantum field theory without these fluctuations [5] and stated only obvious unexplainability and paradoxicality of the situation. The existence of the rotational alternative clearly explains and solves this problem.

Let in the Hamiltonians of the oscillatory $\left(H_{o s c}\right)$ and rotatory $\left(H_{r o t}\right)$ quantization's compare contributions of separate terms to observable effects. An energy 
of free electrons and photons $H_{0}^{(r)}$ and energy of their interactions $H_{I}^{(r)}$ in both cases are identical. Only distinction is that $H_{o s c}$ contains the zero-point vacuum energy $H_{0}^{(0)}$ in the free Hamiltonian and the interaction energy of charges with fluctuating vacuum fields $H_{I}^{(0)}$, while at the rotatory treatment of charge-symmetric system the zero-point fluctuations there absent:

$$
\begin{gathered}
H_{r o t}=\left(H_{0}^{(r)}+H_{I}^{(r)}\right), \\
H_{o s c}=\left(H_{0}^{(r)}+H_{I}^{(r)}\right)+\left[H_{0}^{(0)}+H_{I}^{(0)}\right],
\end{gathered}
$$

Terms in round brackets in (19) and (20), identical in both Hamiltonians, lead to the diagram techniques and in the form of loop contributions of quanta completely explain and with great accuracy describe the Lamb shift and the anomalous magnetic moments as effects from $H_{I}^{(r)}$ only. This means that the rotatory quantization without zero-point contributions is in agreement with these experiments.

At the same time, in the oscillatory approach with $H_{\text {osc }}$, in addition to these contributions, there will be necessarily present also the purely vacuum contributions from $H_{I}^{(0)}$, which, as it is well known, are the same order, as the loop contributions. The sum of these two independent and additive contributions give almost twice more value, than the observable ones (a Fig. 1a, 1b), and thus in fact the oscillatory approach is rejected by these experiments.

In the case of Casimir effect the terms in brackets in (19) and (20) contribute through a fluctuating radiation field of crystal's atoms. As it is well known, the theory of Van der Waals forces, improved by other methods, successfully describes the Casimir effect, including temperature dependences and properties of materials, through radiation of real sources - of atoms - at their zero-point vibrations (i.e. vibrations at zero temperature (!) of the crystal). Since the contributions of purely vacuum fields from $H_{I}^{(0)}$ are the same order and must be added to the contribution from the radiation of atoms ( $\left.H_{I}^{(r)}\right)$, the oscillatory approach in this case gives almost twice more value for the Casimir effect, than observing one and is rejected by these experiments. Rotatory quantization only with contributions of fields of atoms again is in agreement with these experiments.

Thus, if in each effect to try single out a pure contribution of the zero-point fluctuations of vacuum fields, which it would be impossible to explain as approximation of contributions of the virtual quanta or fields of real sources, in each case we find that the purely vacuum contribution is absent [6]. But, as approximations of exact contributions of fields of real sources it is possible to take, of course, effective fluctuating external fields and the results of such approximate calculations are close to the observing data, but it is already really simplified image of the basic and exact picture with fields of real sources.

From observations also follows a practically vanishing (from the particle physics scales) value for the cosmological constant. This fact also cannot be explained in frameworks of the oscillatory quantization. Moreover, the oscillatory treatment predicts the contribution to a cosmological constant of energy of vacuum of already known fields almost in $10^{120}$ time exceeding the observations which has no any meaning. At the same time rotatory quantization predicts lack of the contribution to the cosmological constant from the vacuum energy of fundamental fields and is in agreement with observations. 


\section{The rotatory quantization of a photon field}

Photons with circular polarization and two projections of a helicity form pure states of a photon field at which the Hamiltonian and the helicity operator are diagonal. The helicity operator $\Lambda=\Lambda^{0}$, at lack of an angular momentum, is a time component of divergence on a total angular momentum tensor $\Lambda^{\mu}=\partial_{v} J^{\mu v}$ and is a projection of spin $\mathbf{S}$ to a momentum direction $\mathbf{k}$, i.e. $\Lambda=\mathbf{S} \cdot \mathbf{k} /|\mathbf{k}|$

Photons of opposite helicities are emitted by a rotating dipole and they behave as two states with opposite sign chiral charges. At dipole transitions between rotational levels $\Delta n= \pm 1$ energies of emitted photons are identical and equal to $\Delta E=\hbar \omega$, where $\omega$ is a cyclic rotation frequency. Photons, thus, are similar to quanta of a harmonic rotator at which frequency is angular speed, energy spectrum of rotational levels are equidistant and double degenerate. Further it will be shown that a symmetry between the opposite helicity states leads as well to the lack of a zero-point energy that makes exact the analogy to the harmonic rotator.

For our purposes it is enough to be restricted only to physical degrees of freedom of the photon field in a convenient frame of reference. The revealing physical properties rotational nature of periodicity and lack of the zero-point energy - then will be preserved in other gauges and reference frames also.

Thus, we will direct an axis of spatial coordinates $x^{3}$ along photon's momentum $\mathbf{k}=\left(0,0, k^{3}\right)$ and choose the gauge $A_{3}=0$, having left only transverse physical components of the field vector $A_{1}, A_{2}$. From latter we form a complex field operator $A$ :

$$
A(x)=\frac{1}{\sqrt{2}}\left(A_{1}+i A_{2}\right), \quad A^{*}(x)=\frac{1}{\sqrt{2}}\left(A_{1}-i A_{2}\right) .
$$

Then the Lagrangian and canonical momenta (densities) for the photon field are:

$$
\begin{gathered}
L=\int d^{3} x \partial_{\mu} A^{*} \cdot \partial^{\mu} A . \\
\pi(x)=\partial_{t} A^{*}, \quad \pi^{*}(x)=\partial_{t} A,
\end{gathered}
$$

The Lagrangian is symmetrical under the global phase transformations:

$$
A^{\prime}=e^{i \theta \Lambda} A, \quad A^{* \prime}=e^{-i \theta \Lambda} A^{*}
$$

and corresponding conserved "chiral charge" $\Lambda$ is the helicity. The helicity operator and the free Hamiltonian look like:

$$
\begin{gathered}
\Lambda=i \int d^{3} x\left(A^{*} \pi^{*}-\pi A\right), \\
H=\int d^{3} x\left(\pi^{*} \pi+\nabla A^{*} \nabla A\right) .
\end{gathered}
$$

These two operators become diagonal only for states of photons with circular polarization when the field vector is constant in modulo and rotates around the momentum direction with a constant angular speed. Therefore in the frequency decomposition of fields and sources the frequency means an angular speed of these rotations. At quantization the rotating source emits or absorbs photons carrying away or getting unit angular momentum quantum. As we see, the photon field in the given gauge and frame formally is like a complex scalar field represented as a set of quanta of a harmonic rotator.

At quantization of any system it is necessary to consider that time is divided on small enough intervals $\Delta t_{i}$ during of which trajectories are approximated by smooth 
classical trajectories. In the field theory it is necessary to place system into a box of large volume $V$ and smoothing of field fluctuations in enough small spatial sells $\Delta V_{j}$ in which the field functions should be averaged. The large volume enters an infrared regularization and the sells regularize large momenta. Further we will suppose all integrals as taken by such careful and correct limiting processes.

Field equations and simultaneous commutators of fields look like:

$$
\begin{gathered}
\partial_{\mu} \partial^{\mu} A=0, \quad \partial_{\mu} \partial^{\mu} A^{*}=0 . \\
i\left[\pi(\mathbf{x}, t), A\left(\mathbf{x}^{\prime}, t\right)\right]=\delta_{x x^{\prime}}^{3}, \quad i\left[\pi^{*}(\mathbf{x}, t), A^{*}\left(\mathbf{x}^{\prime}, t\right)\right]=\delta_{x x^{\prime}}^{3}
\end{gathered}
$$

A frequency decomposition of field variables on normal modes of the rotation waves with $k_{0}=\omega_{\mathbf{k}}, \omega_{\mathbf{k}}=|\mathbf{k}|$ gives:

$$
\begin{array}{cc}
A(x)=\sum_{k}\left(a_{k} e^{-i k x}+\beta_{k}^{*} e^{i k x}\right), & A^{*}(x)=\sum_{k}\left(a_{k}^{*} e^{i k x}+\beta_{k} e^{-i k x}\right), \\
\pi(x)=i \sum_{k} \omega_{k}\left(a_{k}^{*} e^{i k x}-\beta_{k} e^{-i k x}\right), & \pi^{*}(x)=-i \sum_{k} \omega_{k}\left(a_{k} e^{-i k x}-\beta_{k}^{*} e^{i k x}\right) .
\end{array}
$$

where the sums are defined as

$$
\sum_{k}=\int \frac{d^{3} k}{\left[(2 \pi)^{3} 2 \omega_{k}\right]^{1 / 2}} .
$$

Here $a_{k}, a_{k}^{*}$ are the annihilation and creation operators of photons of the helicity $\lambda=+1$ and rotation frequency $\omega_{\mathbf{k}}$, and $\beta_{k}, \beta_{k}^{*}$ should correspond to quanta with $\lambda=+1$ , however not directly, but after fulfilling the charge-conjugation symmetry conditions. The commutators of fields (28) lead to following non-zero commutators:

$$
\left[a_{k}, a_{k^{\prime}}^{*}\right]=\delta_{k k^{\prime}}^{3},\left[\beta_{k}, \beta_{k^{\prime}}^{*}\right]=\delta_{k k^{\prime}}^{3}
$$

The helicity operator and the Hamiltonian, by taking into account (30), take a form:

$$
\begin{aligned}
& \Lambda=\int d^{3} k \Lambda_{k}=\int d^{3} k\left(\Lambda_{k}^{(a)}+\Lambda_{k}^{(\beta)}\right)=\int d^{3} k\left(a_{k}^{*} a_{k}-\beta_{k} \beta_{k}^{*}\right), \\
& H=\int d^{3} k H_{k}=\int d^{3} k\left(\Lambda_{k}^{(a)}-\Lambda_{k}^{(\beta)}\right) \omega_{k}=\int d^{3} k\left(a_{k}^{*} a_{k}+\beta_{k} \beta_{k}^{*}\right) \omega_{k} .
\end{aligned}
$$

At a "charge-conjugation" operation ( $C$-conjugation), when a particle transforms to antiparticle (and contrary), in our case changing a sign of the "chiral charge"- the helicity, a "right-rotating" photon (particle) turns to a "left-rotating" photon (antiparticle). There the C-symmetry conditions take the form:

$$
\begin{aligned}
& \Lambda_{k}^{c} \equiv C \Lambda_{k} C^{-1}=-\Lambda_{k}, \\
& H_{k}^{c} \equiv C H_{k} C^{-1}=H_{k},
\end{aligned}
$$

Thus, the operators $a_{k}, a_{k^{\prime}}^{*}$ will be $C$-conjugate, being transformed into the annihilation and creation operators of photons with the helicity $\Lambda=-1$ and similar for $\beta_{k}, \beta_{k}^{*}$ :

$$
\begin{array}{lll}
C a_{k} C^{-1}=b_{k}, & C a_{k}^{*} C^{-1}=b_{k}^{*}, & {\left[b_{k}, b_{k^{\prime}}^{*}\right]=\delta_{k k^{\prime}}^{3},} \\
C \beta_{k} C^{-1}=\alpha_{k}, & C \beta_{k}^{*} C^{-1}=\alpha_{k}^{*}, & {\left[\alpha_{k}, \alpha_{k^{\prime}}^{*}\right]=\delta_{k k^{\prime}}^{3},}
\end{array}
$$

They determine the frequency decomposition of $C$-conjugate field functions:

$$
A^{c}(x)=\sum_{k}\left(b_{k} e^{-i k x}+\alpha_{k}^{*} e^{i k x}\right), \quad A^{c^{*}}(x)=\sum_{k}\left(b_{k}^{*} e^{i k x}+\alpha_{k} e^{-i k x}\right) .
$$


The expressions for the $C$-conjugate helicity and Hamiltonian take a form:

$$
\begin{aligned}
& \Lambda^{c}=\int d^{3} k \Lambda_{k}^{c}=\int d^{3} k\left(\Lambda_{k}^{(b)}+\Lambda_{k}^{(\alpha)}\right)=\int d^{3} k\left(b_{k}^{*} b_{k}-\alpha_{k} \alpha_{k}^{*}\right), \\
& H^{c}=\int d^{3} k H_{k}^{c}=\int d^{3} k\left(\Lambda_{k}^{(b)}-\Lambda_{k}^{(\alpha)}\right) \omega_{k}=\int d^{3} k\left(b_{k}^{*} b_{k}+\alpha_{k} \alpha_{k}^{*}\right) \omega_{k} .
\end{aligned}
$$

Now requirements of $C$-symmetry for field modes we write in details:

$$
\begin{aligned}
& \Lambda_{k}=\Lambda_{k}^{(a)}+\Lambda_{k}^{(\beta)}=-\left(\Lambda_{k}^{(b)}+\Lambda_{k}^{(\alpha)}\right)=-\Lambda_{k}^{c} \\
& H_{k}=\omega_{k}\left(\Lambda_{k}^{(a)}-\Lambda_{k}^{(\beta)}\right)=\omega_{k}\left(\Lambda_{k}^{(b)}-\Lambda_{k}^{(\alpha)}\right)=H_{k}^{c},
\end{aligned}
$$

or in a compact form:

$$
\begin{aligned}
& \Lambda_{k}^{(a)}+\Lambda_{k}^{(\beta)}=-\Lambda_{k}^{(b)}-\Lambda_{k}^{(\alpha)}, \\
& \Lambda_{k}^{(a)}-\Lambda_{k}^{(\beta)}=\Lambda_{k}^{(b)}-\Lambda_{k}^{(\alpha)} .
\end{aligned}
$$

Combining and subtracting these two operator relations, we obtain:

$$
\Lambda_{k}^{(\beta)}=-\Lambda_{k}^{(b)}, \quad \Lambda_{k}^{(\alpha) p}=-\Lambda_{k}^{(a)} .
$$

or in the explicit form:

$$
\beta_{k} \beta_{k}^{*}=-b_{k}^{*} b_{k}, \quad \alpha_{k} \alpha_{k}^{*}=-a_{k}^{*} a_{k} .
$$

By substituted it into (33) and (37), we obtain the final expressions for the observables:

$$
\begin{gathered}
\Lambda=-\Lambda^{c}=\int d^{3} k\left(\Lambda_{k}^{(a)}+\Lambda_{k}^{(b)}\right)=\int d^{3} k\left(a_{k}^{*} a_{k}-b_{k}^{*} b_{k}\right) . \\
H=H^{c}=\int d^{3} k\left(\Lambda_{k}^{(a)}-\Lambda_{k}^{(b)}\right) \omega_{k}=\int d^{3} k\left(a_{k}^{*} a_{k}+b_{k}^{*} b_{k}\right) \omega_{k} .
\end{gathered}
$$

Thus, observables are expressed through the helicity operators $\Lambda_{k}^{(a)}$ and $\Lambda_{k}^{(b)}$, constructed from the creation-annihilation operators of photons of opposite helicity. In particular, at $n_{a}=0, n_{b}=0$ the annihilation operators define the vacuum: $a_{k}|0\rangle=0$, $b_{k}|0\rangle=0$, which according to (43) does not contain a zero-point energy. The helicity of the vacuum also is equal to zero. All this is natural for the field states analogous to the harmonic rotator states.

Thus, quantization of the photon field with circular polarization is related to quantization of normal modes of the field as harmonic rotators. The ground state energy, thus, as in the systems where purely rotational modes are quantized, does not contain the zero-point energy.

Thus, quantization of transverse (physical) degrees of freedom of the photon field is similar to quantization of a complex scalar field. Photons of opposite helicities behave as a particle and an antiparticle. Because of invariance of properties of vacuum the fact of lack of the zero-point energy of vacuum, established in one a frame of reference and in one a gauge, is valid for all frames of reference and for all gauges.

The above presented formalism of quantization of the photon field in the source's rest frame, it is easy to put a relativistically-covariant form, preserving the $C$-symmetry of the system. Transition to other frames of reference can be realized by the tetrad vectors: 


$$
\begin{aligned}
& A_{1}=e_{1}^{\mu} A_{\mu}, \quad A_{2}=e_{2}^{\mu} A_{\mu}, \quad e_{0}^{\mu} A_{\mu}=e_{3}^{\mu} A_{\mu}=0, \\
& A(x)=\frac{1}{\sqrt{2}}\left(e_{1}^{\mu}+i e_{2}^{\mu}\right) A_{\mu}=e_{+}^{\mu} A_{\mu}, \\
& A^{*}(x)=\frac{1}{\sqrt{2}}\left(e_{1}^{\mu}-i e_{2}^{\mu}\right) A_{\mu}=e_{-}^{\mu} A_{\mu}=e_{+}^{\mu^{*}} A_{\mu} .
\end{aligned}
$$

Then, the photon field, having two transverse components $\left(0, A_{1}, A_{2}, 0\right)$ in the rest frame system of the source, after the Lorentz transformation to other frames of reference in general has all four components $A_{\mu}=e_{\mu}^{1} A_{1}+e_{\mu}^{2} A_{2}$. The longitudinal component appears simply due to another orientation of spatial axes of the new frame. The time component appears at the Lorentz transformation of projections $\left(A_{1}, A_{2}\right)$ to a direction of motion of the new frame of reference. Propagators of photons will include covariant expressions:

$$
e_{\mu}^{-} e_{v}^{+} A\left(\Lambda x^{\prime}\right) A^{*}(\Lambda x)=e_{\mu}^{a} e_{v}^{b} A_{a}\left(\Lambda x^{\prime}\right) A_{b}(\Lambda x)=A_{\mu}\left(x^{\prime}\right) A_{\nu}(x) .
$$

Since the tetrad vectors $\left(e_{\mu}^{1}, e_{\mu}^{2}\right)$ are constant and purely kinematical factors, they lead only to kinematical corrections to the quantization procedure and do not change main physical consequences.

\section{Gauge fields and gravitons}

At quantization of a photons field a physical nature of frequencies of quanta has appeared related to rotations of field vectors and this fact has led to the theory without a vacuum zero-point energy in accordance with experiments. Since the formal structure and properties of relativistic fields are mainly similar, the same result can be expected and for other fields also. Therefore turning to systematic study of fields of different spins, we will consider at first analogues of photons - quanta of non-Abelian gauge fields and gravitons.

Quantization of non-Abelian gauge fields $A_{\mu}^{a}$ (spin 1) and graviton field (spin 2) in a weak field limit $g^{2} \ll 1$ are reduced to quantization of two transverse physical degrees of freedom of several vector or tensor fields. The massless gauge fields have an axial symmetry and at circular polarization their free Hamiltonians and helicity operators are diagonal. Therefore, the above formulated rotatory quantization of a photon field is applicable to this case also. Taking into account of non-linearity and internal symmetries of these fields does not change main conclusions about a rotational nature of their frequencies and lack of a zero-point energy in ground states of their free fields.

The non-linear contributions are proportional to the coupling constant $g$ both in the self-interaction terms and the covariant derivatives. Therefore, at the weak field approximation we can neglect these terms and quantize the gauge fields and the graviton field as sets of independent photon degrees of freedom.

In the case of the graviton field an effective coupling constant is weak up to the Planck energy where the gravitational radius of a particle becomes equal to its wavelength and appears a strong gravitational redshift of frequencies of quantum fluctuations. Rapidly decreasing of frequencies at the Planck length because of freezing of proper times with respect to the "distant observer's time", leads to the negligibility and insufficiency of contribution of nonlinear terms.

Lack of a zero-point energy of modes of a free field follows from the rotational nature of periodicity and frequencies of sources and their quanta and, consequently, the additional interactions cannot change it. All contributions to the vacuum energy density 
from interactions will be proportional to their coupling constants and disappear in the weak field limit (taking into account the cutting at the Planck length), whereas a true zero-point energy does not depend on these factors.

The conclusion that at rotatory quantization of gauge fields and graviton field in one a frame of reference, in the transverse gauge and in the weak-field approximation the zero-point vacuum energy disappears, holds for all frames of reference and for all gauges because of invariance of properties of the vacuum.

All these, of course, concern to systems without vacuum condensates (or at least regions without them) and to situations where non-perturbative effects do not dominate. Contributions of condensates and topologically nontrivial solutions in the vacuum energy will be considered in forthcoming publication, but they also obviously do not change the conclusions concerning the free fields.

\section{Complex scalar and vector fields}

At quantization of rotations of a complex scalar and vector fields, unlike of photons at which field vectors rotate in the real space, it is necessary to study rotations in a complex space of field variables - in an isotopic space.

The Lagrangian of the scalar field $\phi=\phi_{1}+i \phi_{2}$ in the minimal form:

$$
L=\int d^{3} x\left(\partial_{\mu} \phi^{*} \cdot \partial^{\mu} \phi-m^{2} \phi^{*} \phi\right),
$$

leads to the canonical momenta, Hamiltonian and charge operator:

$$
\begin{gathered}
\pi(x)=\partial_{t} \phi^{*}, \quad \pi^{*}(x)=\partial_{t} \phi \\
H=i \int d^{3} x\left(\pi \pi^{*}+\nabla \phi^{*} \nabla \phi+m^{2} \phi^{*} \phi\right) . \\
Q=i \int d^{3} x\left(\phi^{*} \pi^{*}-\pi \phi\right)
\end{gathered}
$$

For free fields there are no problems with ordering of operators, but at inserting of interaction with gauge fields the charge operator depends on ordering.

The general solution of field equations includes the frequency modes with a 4momentum, $k=\left( \pm \omega_{\mathbf{k}}, \mathbf{k}\right) \omega_{\mathbf{k}}=\left(\mathbf{k}^{2}+m^{2}\right)^{1 / 2}$, but then negative-frequency modes can be replaced to positive-frequency antiparticles. Since all formalism is similar to the case of a photon field (with addition of mass) there appears a problem on, how we can consider $\omega_{\mathbf{k}}$ as a rotation frequency. In the system described by a complex field $\phi=\phi_{1}+i \phi_{2}$ with the Lagrangian (46) there is a global $U(1)$ symmetry under rotation on angle $\lambda$ in twodimensional field space $\left(\phi_{1}, \phi_{2}\right)$ :

$$
\phi^{\prime}(x)=e^{i \lambda Q} \phi(x), \phi^{*}(x)=e^{-i \lambda Q} \phi^{*}(x) .
$$

By analogy with the photon field, for the scalar field also, represented as an isovector in two-dimensional field space $\vec{\phi}=\left(\phi_{1} \vec{n}_{1}, \phi_{2} \vec{n}_{2}\right)$, we restrict us by field model only with those modes, when this isovector rotates with frequency $\omega_{k}$, periodically coinciding by $\phi_{1}$ or $\phi_{2}$. Such rotation of field's isovector, generated by the source, in real space then propagates as field's quantum.

In a complex representation the decomposition of such field on the normal modes has the same form, as (29) - (30) with replacement $A \rightarrow \phi$ and practically with the same formalism. Quantization of normal modes of these rotational waves also will lead to the opposite charged quanta of the complex scalar field. The charge in this case, as an 
analogue of the helicity, is a projection of an isospin to a normal to an isotopic plane and is defined (up to a constant) by the third component of the isospin: $Q \sim \tau_{3}$.

Thus, in the rotatory quantization isospin of the complex scalar field is interpreted as the angular momentum of rotations in isotopic plane with frequency of the field quantum. Quantization of the charge, which is "isotopic helicity", and energy appear then as the consequences of isovector's angular momentum quantization.

To two equivalent rotation directions in field space here correspond two signs of the charge and there take place a charge conjugation symmetry ( $C$-symmetry). At $C$ conjugation the Hamiltonian is invariant, while the charge operator changes a sign:

$$
H^{c} \equiv C H C^{-1}=H, \quad Q^{c} \equiv C Q C^{-1}=-Q .
$$

This leads to charge-conjugate ladder operators:

$$
b_{k}=C a_{k} C^{-1}, \quad b_{k}^{*}=C a_{k}^{*} C^{-1},
$$

which define $C$-conjugate vacuum $b_{k}\left|0_{b}\right\rangle=0$ and the excited states $\left|n_{b}\right\rangle$. The observables $Q^{c}$ and $H^{c}$ also are expressed through them.

Further starting from the frequency decomposition of the field on the rotational modes:

$$
\phi(x)=\sum_{k}\left(a_{k} e^{-i k x}+\beta_{k}^{*} e^{i k x}\right), \quad \phi^{*}(x)=\sum_{k}\left(a_{k}^{*} e^{i k x}+\beta_{k} e^{-i k x}\right),
$$

and applying the same method, as for the photon field, we obtain:

$$
\begin{aligned}
& Q=\int d^{3} k\left(a_{k}^{*} a_{k}-\beta_{k} \beta_{k}^{*}\right)=\int d^{3} k\left(a_{k}^{*} a_{k}-b_{k}^{*} b_{k}\right), \\
& H=\int d^{3} k\left(a_{k}^{*} a_{k}+\beta_{k} \beta_{k}^{*}\right) \omega_{k}=\int d^{3} k\left(a_{k}^{*} a_{k}+b_{k}^{*} b_{k}\right) \omega_{k} .
\end{aligned}
$$

Thus, the operators observables of the complex scalar field with exact $C$-symmetry are normal-ordered and the energy and charge of the vacuum vanish.

A complex vectors field's momentum decomposition to rotational modes is the same as for a scalar field, but by including the polarization vector $\varepsilon_{\mu k}^{\lambda}$ :

$$
\begin{aligned}
& B_{\mu}(x)=\sum_{\mathbf{k} \lambda}\left(a_{k \lambda} \varepsilon_{\mu k}^{\lambda} e^{-i k x}+\beta_{k \lambda}^{*} \varepsilon_{\mu k}^{\lambda^{*}} e^{i k x}\right), \\
& B_{\mu}^{*}(x)=\sum_{\mathbf{k} \lambda}\left(a_{k \lambda}^{*} \varepsilon_{\mu k}^{\lambda^{*}} e^{i k x}+\beta_{k, \lambda} \varepsilon_{\mu k}^{\lambda} e^{-i k x}\right) .
\end{aligned}
$$

After selecting of independent degrees of freedom, the field equations for three polarizations remain linear and are quantized in any frame of reference independently as three complex scalar fields. Therefore, dropping details, we can present only results for the Hamiltonian and the charge operator of the complex vector field:

$$
\begin{aligned}
& Q=\sum_{\lambda} \int d^{3} k\left(a_{k \lambda}^{*} a_{k \lambda}-\beta_{k \lambda} \beta_{k \lambda}^{*}\right)=\sum_{\lambda} \int d^{3} k\left(a_{k \lambda}^{*} a_{k \lambda}-b_{k \lambda}^{*} b_{k \lambda}\right), \\
& H=\sum_{\lambda} \int d^{3} k\left(a_{k \lambda}^{*} a_{k \lambda}+\beta_{k \lambda} \beta_{k \lambda}^{*}\right) \omega_{k}=\sum_{\lambda} \int d^{3} k\left(a_{k \lambda}^{*} a_{k \lambda}-b_{k \lambda}^{*} b_{k \lambda}\right) \omega_{k},
\end{aligned}
$$

which also do not contain the zero-point energy and zero-point charge.

Notice, that although this result is obtained in a fixed frame of reference, nevertheless, the fact of lack of the zero-point energy and zero-point charge does not depend on a frame of reference since the vacuum energy-momentum tensor $\Lambda g_{i k}$ is proportional to a scalar constant $\Lambda$ and if this constant vanishes in one a frame, it disappears in all others also. 
The symmetries of complex fields allow only gauge-invariant interactions of the form $\left(\phi^{*} \phi+\phi_{c}^{*} \phi_{c}\right)^{n}$, conserving a charge. At rotatory quantization, on the one hand, energies of quanta are determined by charge operators, and on the other hand, interactions of quanta lead to redistribution of these charges. Since the charge operator normalordered in all orders of the perturbation theory, any degree of this operator also will be normal-ordered, then the vacuum matrix elements from them disappear. This fact sufficiently reduces a number of diagrams in all orders of the perturbation theory. Such contributions, and also loop diagrams will be considered in further publications.

\section{Spinor fields}

The rotational treatment of photon's frequency allows us come to a rotational treatment of frequencies of fermions and their spin also, which from the physical point of view looks rather attractively. Here we will formulate the rotatory quantization formalism for the spinor field without detailed physical interpretation.

The standard Lagrangian, Hamiltonian and charge operator of the spinor field are:

$$
\begin{aligned}
L & =\int d^{3} x\left[\bar{\psi} \gamma^{\mu}\left(i \partial_{\mu} \psi\right)-m \bar{\psi} \psi\right], \\
H & =\int d^{3} x \psi^{+}\left(i \partial_{t} \psi\right)-\int d^{3} x\left(i \partial_{t} \psi^{+}\right) \psi, \\
Q & =\int d^{3} x \psi^{+} \psi .
\end{aligned}
$$

The frequency decomposition of the spinor field on rotational modes looks like:

$$
\begin{aligned}
& \psi(x)=m \sqrt{2} \sum_{\alpha p}\left(b_{p \alpha} u_{p}^{\alpha} e^{-i p x}+\beta_{p \alpha}^{+} v_{p}^{\alpha} e^{i p x}\right), \\
& \psi^{+}(x)=m \sqrt{2} \sum_{\alpha p}\left(b_{p \alpha}^{+} u_{p}^{\alpha+} e^{i p x}+\beta_{p \alpha} v_{p}^{\alpha+} e^{-i p x}\right),
\end{aligned}
$$

where the normalization is $u_{p}^{\alpha+} u_{p}^{\alpha^{\prime}}=v_{p}^{\alpha+} v_{p}^{\alpha^{\prime}}=\delta^{\alpha \alpha^{\prime}} E_{p} / m$ and $E_{p}=\left(\mathbf{p}^{2}+m^{2}\right)^{1 / 2}$.

As usual, the spinor fields are quantized through the equal time anticommutators:

$$
\begin{aligned}
& \left\{\bar{\psi}(\mathbf{x}, t), \psi\left(\mathbf{x}^{\prime}, t\right)\right\}=\delta_{x x^{\prime} I_{(4)},} \\
& \left\{\psi(\mathbf{x}, t), \psi\left(\mathbf{x}^{\prime}, t\right)\right\}=\left\{\bar{\psi}(\mathbf{x}, t), \bar{\psi}\left(\mathbf{x}^{\prime}, t\right)\right\}=0,
\end{aligned}
$$

which lead to the anticommutators for each mode, non-zero of which are:

$$
\left\{b_{p \alpha}, b_{p \alpha}^{+}\right\}=\left\{\beta_{p \alpha}, \beta_{p \alpha}^{+}\right\}=\delta_{p p^{\prime}}^{3} \delta_{\alpha \alpha^{\prime}} .
$$

Then, for the charge operator and Hamiltonian of the free spinor field we obtain:

$$
\begin{aligned}
& Q=\sum_{\alpha} \int d^{3} p\left(Q_{p \alpha}^{(b)}+Q_{p \alpha}^{(\beta)}\right)=\sum_{\alpha} \int d^{3} p\left(b_{p \alpha}^{+} b_{p \alpha}+\beta_{p \alpha} \beta_{p \alpha}^{+}\right), \\
& H=\sum_{\alpha} \int d^{3} p\left(Q_{p \alpha}^{(b)}-Q_{p \alpha}^{(\beta)}\right) E_{p}=\sum_{\alpha} \int d^{3} p\left(b_{p \alpha}^{+} b_{p \alpha}-\beta_{p \alpha} \beta_{p \alpha}^{+}\right) E_{p} .
\end{aligned}
$$

By introducing charge-conjugate ladder operators:

$$
\begin{array}{ll}
C b_{p \alpha} C^{-1}=d_{p \alpha}, & C b_{p \alpha}^{+} C^{-1}=d_{p \alpha}^{+}, \\
C \beta_{p \alpha} C^{-1}=\alpha_{p \alpha}, & C \beta_{p \alpha}^{+} C^{-1}=\alpha_{p \alpha}^{+}
\end{array}
$$

we obtain the charge-conjugate to (61) expressions for the observables: 


$$
\begin{aligned}
& Q^{c}=\sum_{\alpha} \int d^{3} p\left(Q_{p \alpha}^{(d)}+Q_{p \alpha}^{(\alpha)}\right)=\sum_{\alpha} \int d^{3} p\left(d_{p \alpha}^{+} d_{p \alpha}+\alpha_{p \alpha} \alpha_{p \alpha}^{+}\right), \\
& H^{c}=\sum_{\alpha} \int d^{3} p\left(Q_{p \alpha}^{(d)}-Q_{p \alpha}^{(\alpha)}\right) E_{p}=\sum_{\alpha} \int d^{3} p\left(d_{p \alpha}^{+} d_{p \alpha}-\alpha_{p \alpha} \alpha_{p \alpha}^{+}\right) E_{p},
\end{aligned}
$$

The $C$-symmetry conditions for each of independent modes look like:

$$
H_{p \alpha}^{c} \equiv C H_{p \alpha} C^{-1}=H_{p \alpha}, \quad Q_{p \alpha}^{c} \equiv C Q_{p \alpha} C^{-1}=-Q_{p \alpha} .
$$

Written in more detail form:

$$
\begin{aligned}
& Q_{p \alpha}=Q_{p \alpha}^{(b)}+Q_{p \alpha}^{(\beta)}=-\left(Q_{p \alpha}^{(d)}+Q_{p \alpha}^{(\alpha)}\right)=-Q_{p \alpha}^{c}, \\
& H_{p \alpha}=\left(Q_{p \alpha}^{(b)}-Q_{p \alpha}^{(\beta)}\right) E_{p}=\left(Q_{p \alpha}^{(d)}-Q_{p \alpha}^{(\alpha)}\right) E_{p}=H_{p \alpha}^{c}
\end{aligned}
$$

they lead to the operator identities:

$$
\begin{array}{cl}
Q_{p \alpha}^{(\beta)}=-Q_{p \alpha}^{(d)}, & Q_{p \alpha}^{(\alpha)}=-Q_{p \alpha}^{(b)}, \\
\alpha_{p \alpha} \alpha_{p \alpha}^{+}=-d_{p \alpha}^{+} d_{p \alpha}, & \beta_{p \alpha} \beta_{p \alpha}^{+}=-b_{p \alpha}^{+} b_{p \alpha} .
\end{array}
$$

Their substitution in (61) and (63) give us the final expressions for $H$ and $Q$, containing the normal-ordered products of the ladder operators:

$$
\begin{aligned}
& Q=-Q^{c}=\sum_{\alpha} \int d^{3} p\left(b_{p \alpha}^{+} b_{p \alpha}-d_{p \alpha}^{+} d_{p \alpha}\right), \\
& H=H^{c}=\sum_{\alpha} \int d^{3} p\left(b_{p \alpha}^{+} b_{p \alpha}+d_{p \alpha}^{+} d_{p \alpha}\right) E_{p} .
\end{aligned}
$$

Notice that the vacuum expectations both from the charge operator and spatial components of a current now disappear:

$$
\langle 0|Q(x)| 0\rangle=0, \quad\langle 0|\mathbf{j}(x)| 0\rangle=0 .
$$

Thus, at quantization of the charge conjugation symmetric modes of the spinor field the zero-point energy and zero-point charge of the vacuum do not arise and the observables are naturally normal-ordered.

\section{Crossing symmetry of states and the causal propagators}

In the previous paper [2] the time reversal and crossing symmetry in complex systems have been considered and the results mainly remain valid for the relativistic fields also. In the relativistic theory a symmetry under 4-inversion $(P T)$ holds in a combination with $C$-symmetry ( $C P T)$, there appears a crossing symmetry of amplitudes also.

A commutator function of a complex scalar field:

$$
\left[\phi\left(x^{\prime}\right), \phi^{*}(x)\right]=i D\left(x^{\prime}-x\right) \text {. }
$$

is standard and, as an even function of the time interval, disappears outside the light cone.

However, a vacuum expectation from $\phi^{*}(x) \phi\left(x^{\prime}\right)$ formally disappears

$$
\left\langle 0\left|\phi^{*}(x) \phi\left(x^{\prime}\right)\right| 0\right\rangle \sim\left\langle 0\left|\beta \beta^{*}\right| 0\right\rangle b=\left\langle 0\left|b^{*} b\right| 0\right\rangle=0,
$$

as a normal-ordered form of the product. Due to this property, and taking into account (70), the vacuum expectation from $\phi\left(x^{\prime}\right) \phi^{*}(x)$ is reduced to the commutator function (70) (see [2]).

However, quantization by means of the auxiliary operators has shown [2], that if we take into account the crossing-symmetry, at last there remain usual operators of the charge-conjugate quanta without contributions of zero-point fluctuations. There the 
contributions of antiparticles may be obtained directly by the charge conjugation of contributions of particles.

Thus, to find the propagators of particles and antiparticles, we can simplify the situation and use the symmetry properties: (1) restrict the model only to the positivefrequency contributions, 2) obtain the propagators for particles, 3) contributions of the antiparticles then obtain by the charge conjugation of the contributions of particles, but with taking into account the crossing symmetry and the causal restrictions.

These steps give directly the standard "causal" propagator:

$$
\begin{aligned}
& \left\langle 0\left|\phi_{+}\left(x^{\prime}\right) \phi_{+}^{*}(x)\right| 0\right\rangle \theta\left(t^{\prime}-t\right)+\left\langle 0\left|\phi_{+}^{c}(x) \phi_{+}^{c^{*}}\left(x^{\prime}\right)\right| 0\right\rangle \theta\left(t-t^{\prime}\right)=i D_{c}\left(x^{\prime}-x\right), \\
& i D_{c}\left(x^{\prime}-x\right)=\sum_{k}\left[\theta\left(t^{\prime}-t\right) e^{-i \omega_{k}\left(t^{\prime}-t\right)}+\theta\left(t^{\prime}-t\right) e^{i \omega_{k}\left(t^{\prime}-t\right)}\right] e^{i \mathbf{k}\left(\mathbf{x}^{\prime}-\mathbf{x}\right)},
\end{aligned}
$$

which do not disappear outside the light-cone.

\section{Conclusion}

At the rotatory quantization of the fields the creation-annihilation operators in the expressions for observables of fields are automatically normal-ordered, the zero-point energy and zero-point charge of the vacuum do not arise. At including of interactions, in the first order the vacuum energy, because of normal-ordered operators, also vanishes. Therefore the reformulation of the covariant perturbation theory with transition from harmonic oscillator quanta to quanta of the harmonic rotator is reduced to a "strong" normal ordering of the Hamiltonians and currents.

Thus, the rotatory quantization of relativistic fields at a final point mainly corresponds to the former standard treatment and, therefore, the agreement with most of experiments is preserved. In certain cases, where the former theory contradicted to the observations due to the zero-point fluctuations, the accordance in the new treatment is reached due to the lack of such fluctuations. Modifications in the standard formalism are minimal and do not break the agreement between the theory and experiment.

Spin of the gauge bosons appears as a generalized angular momentum relating to rotations of field vectors in real space whereas in case of internal symmetries isospins (charges) are the generalized angular momenta at rotations of field vectors in a field space.

The rotatory quantization of relativistic fields allows us to understand the physical nature of a spin and charges of particles on a unified basis, relating them with the angular momenta at rotations of field vectors with the frequency, defining energy of quanta. Moreover, quantized nature of energy, spin and charges of quanta appears as a result of the quantized nature of the angular momenta of the field vectors.

\section{References}

1. Zakir Z. (2011) Theor. Phys., Astrophys. and Cosmol., 6, 1, 1.

2. Zakir Z. (2011) Theor. Phys., Astrophys. and Cosmol., 6, 2, 14. 6, 2, 31 .

3. Zakir Z. (2006) Theor. Phys., Astrophys. and Cosmol., 1, 1, 11. 1, 4, 61 . 